\title{
Functional results of surgical treatment for acromioclavicular joint dislocation using the modified weaver-dunn surgical technique
}

\begin{abstract}
Objective: To evaluate the functional outcome of patients who underwent surgical treatment using the modified Weaver-Dunn technique for the treatment of acute acromioclavicular dislocation.

Method: Retrospective case series study (level of evidence IV) based on their view of medical records of 20 patients who participated in a 25 -week postoperative follow-up, in which they were assessed using the UCLA score and submitted to radiological control. This follow-up was due to surgical treatment for acromioclavicular dislocation using the modified Weaver-Dunn technique, with two $5.5 \mathrm{~mm}$-Peek Zip ${ }^{\circledR}$ anchors fixed to the coracoid process, with ties on the clavicle, and transfer of the coracoacromial ligament to the distal clavicle.
\end{abstract}

Results: The patients were followed-up for 25 weeks, and all presented satisfactory functional results, with $70 \%$ considered excellent and $30 \%$ classified as good. However, a high rate of reduction loss of the acromioclavicular joint was observed, which corresponded to 6 of the 20 cases monitored. The average time before returning to routine activities was 20.7 weeks; the shortest was 16 weeks, and the longest, 30 weeks.

Conclusion: In the present study, we found that all patients who were submitted to the described technique presented a low level of morbidity and satisfactory functional results (excellent and good), successfully returning to their everyday activities.
Volume 12 Issue 2 - 2020

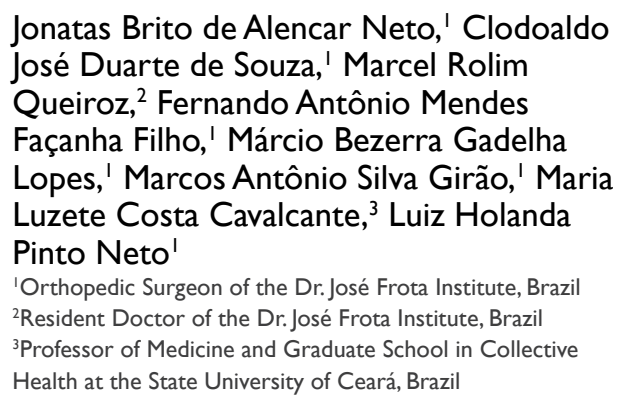

Correspondence: Jonatas Brito de Alencar Neto, Orthopedic Surgeon of the Dr. José Frota Institute, 1816 Barão do Rio Branco Road, Fortaleza, Ceará, Brazil, Tel +5585999561797, Email Jonatasbrito19@hotmail.com

Received: March 24, 2020 | Published: April 28, 2020

Keywords: acromioclavicular joint, joint dislocations, rehabilitation

\section{Introduction}

Acromioclavicular joint injuries are prevalent among the disorders caused by trauma, corresponding to around $9 \%$ of all injuries involving the shoulder girdle,,$^{1,2}$ with a total of approximately 1.8 people per 10,000 inhabitants sustaining the injury per year. The primary risk factors include contact-involving sports activities and automobile accidents, which explains its higher incidence among young adult males. ${ }^{3}$

A fundamental principle in the treatment of joint injuries is to restore congruence, aiming to decrease the incidence of arthritis. Many acromioclavicular joint injuries receive conservative treatment, varying from the use of a simple shoulder sling to a splint or cast, while others require surgery.

The Rockwood classification for acromioclavicular dislocation, which varies from types I to VI, has been widely utilized. Overall, there is an agreement in which injuries type I and II must be treated conservatively, while types IV, V, and VI are treated surgically. However, there has been considerable discussion comparing the conservative method of treatment with the surgical one regarding type-III injuries, with several studies conducted on the issue but with no consensus on the ideal choice. ${ }^{4-9}$

There are more than 200 surgical techniques, ${ }^{10}$ and most of those that aim at joint fixation and reconstruction of the coracoclavicular ligament involve the use of metal implants, including Kirschner wires,
Steinmann pins, hook plate fixation, and Bosworth screws. One of the most employed and widespread techniques for surgical treatment is the modified Weaver-Dunn procedure, which involves the transfer of the coracoacromial ligament and its insertion in the acromion to the distal third of the clavicle with the aid of ties. ${ }^{11-13}$

The objective of the present study was to evaluate the functional results of patients who underwent surgical treatment using the modified Weaver-Dunn technique for the treatment of acromioclavicular dislocation, with the UCLA (University of California, Los Angeles) score system. ${ }^{14,15}$ It is understood that this technique will lead to a good functional outcome, returning the same working function prior to injury.

\section{Materials and methods}

This retrospective study comprised the review of medical records of 32 patients diagnosed with acromioclavicular dislocation, who underwent surgery using the modified Weaver-Dunn technique by the Orthopedics and Traumatology service of a tertiary referral hospital in northeastern Brazil between April 2011 and January 2016. The preoperative evaluation of such patients was carried out using radiographs AP shoulder radiograph of the ipsilateral and contra lateral sides of the injury.

The inclusion criteria were: ages between 18 and 70, Rockwood classification of type-III injury or higher, use of the modified WeaverDunn technique, and an outpatient follow-up of at least 24 weeks. 
Exclusion criteria included previous injury of the operated shoulder, more than one fracture in the same shoulder, follow-up interruption, or lack of sufficient data in the medical records.

The minimum follow-up time was 06 months, with follow-up appointments after 2, 6, 12, and 25weeks.After surgery, the operated limb was immobilized in a Velpeau sling for six weeks. As part of the rehabilitation program, pendulum movements and internal and external rotations at zero degrees were authorized immediately after surgery. Passive and active movements (to gain range of motion) started six weeks after the procedure. Each follow-up appointment consisted of a radiological control similar to that in the preoperative stage (Figure 1) and physical examinations. The medical team chose the UCLA score as the criterium for evaluation due to its practicality and the inclusion of the patient's satisfaction criterium, with the results being interpreted using Ellman criteria.

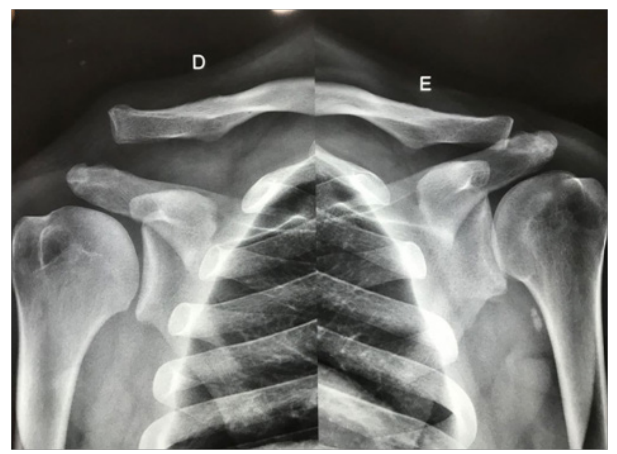

Figure I Preoperative radiograph of both shoulders.

The University of California, Los Angeles (UCLA) shoulder score was developed in 1981, prior to the routine utilization of modern psychometrics. Consequently, the methods employed in its development are not explained, including question elaboration and weighting. The score consists of a combination of physical exam findings (active forward elevation and strength) and subjective patient-reported measures (pain, satisfaction, and function). Pain and functionality are preferentially weighted (20 out of 35 possible points), with higher scores indicating better function. The UCLA score has been utilized to assess a variety of shoulder conditions. Limitations of the scoring method are inherent to its design. Many of the questions are double-barreled, meaning that multiple inquiries are combined within a single question. For example, the pain scale responses address both frequency of pain and type of analgesia Respondents may present difficulty selecting an appropriate response to the question since they support only a portion of one selection, not the entire response. Furthermore, the satisfaction portion of the instrument only allows the UCLA score to be logically used postintervention, hindering the determination of responsiveness. Similar to the Constant score, including both the physical exam and patient self-assessment renders the UCLA multi-dimensional, meaning that it combines multiple domains into a single score..$^{14,15}$ This study was approved by the Institutional Review Board, under process number 57205616.7.0000.5047.

\section{Surgical technique}

The surgical procedure was conducted with the patient under brachial plexus block, associated with general anesthesia. The operating table was arranged in the "beach chair position" with the patient in supine position, and a pillow was placed under the scapula of the injured side. Asepsis and antisepsis were performed, followed by the apposition of sterile fields. Next, a slightly oblique incision was made from the posterior distal third of the clavicle, near the acromioclavicular joint, toward the anterior coracoid process, following Langer's lines. The delto-trapezial fascia was transversely sectioned, and the deltoid and trapezius muscles excised from the clavicle and the acromion $(1 \mathrm{~cm})$. From the base of the coracoid process, the coracoacromial ligament was identified down to its acromial origin. Excision of the ligament from the acromion was performed using an osteotome, aiming at having the maximum length in a small block of bone. Next, ligament repair was conducted with braided silicon polyester suture (Ethibond ${ }^{\circledR}$ ). Then, two $5.5 \mathrm{~mm}$-Peek Zip ${ }^{\circledR}$ (Stryker, Kalamazzo, MI, USA) suture anchors were fixed to the coracoid process. Afterward, three holes were drilled, with a $2.5 \mathrm{~mm}$ drill, into the distal end of the clavicle; the holes are arranged in a triangular format, with a basis for the distal medial and apex. Excision of the distal end of the clavicle is carried out in chronic cases and in acute cases with arthritis. The anchor thread is passed through the most medial holes, and the thread for coracoacromial ligament repair has one end passed through the most lateral hole, and the other, through the anteromedial hole. The entire thread is passed through the clavicle in caudal-cranial direction. The clavicle is then reduced by aligning its upper cortex with that of the acromion, and the thread ends are tied. The delto-trapezial fascia is always carefully sutured with orthopedic Vicryl ${ }^{\circ}$ 2, since we believe it is an additional factor for the horizontal stability of the acromioclavicular joint. No procedures were performed on the acromioclavicular ligaments (Figure 2).

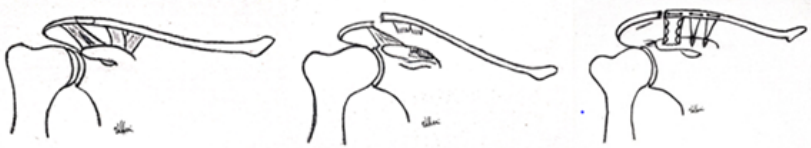

Figure 2 Schematic representation of the modified Weaver-Dunn surgical technique.

\section{Results}

Only 20 of the patients completed the postoperative followup of 25 weeks and, therefore, were included in the study. Eleven were classified as type III and nine as type-V injury, according to the Rockwood classification. All patients in the study were operated on by the same shoulder specialist.

Seventeen patients were male and three were female. The average age was 42.75 years, varying from 23 to 66 years of age, and all of them were right-handed. Six of the patients had injuries on the left shoulder, and fourteen, on the right. As for the trauma mechanism, nine were caused by motorcycle accidents, ten by falling to the ground, and one by a higher than 1-meter fall.

The average time before returning to usual activities was 20.7 weeks, the shortest being 16 weeks, and the longest, 30 weeks. The radiological control for contralateral comparison regarding the coracoclavicular distance showed similar or lower level in 14 patients, elevation lower than $50 \%$ in three, between 50 and $100 \%$ in two, and higher than $100 \%$ in one patient.

The UCLA score was applied at 6,12 , and 25 weeks. The average number of points after six weeks was 21.7; after 12 weeks, 29.95; and after 25 weeks, 33.95, with variation between 30 and 35. After 25 weeks (when the UCLA score was last applied in the follow-up), 
14 patients had 35 points, classified as an excellent result according to the Ellman criteria, and six had between 30 and 33 points, classified as good (Figure 3).

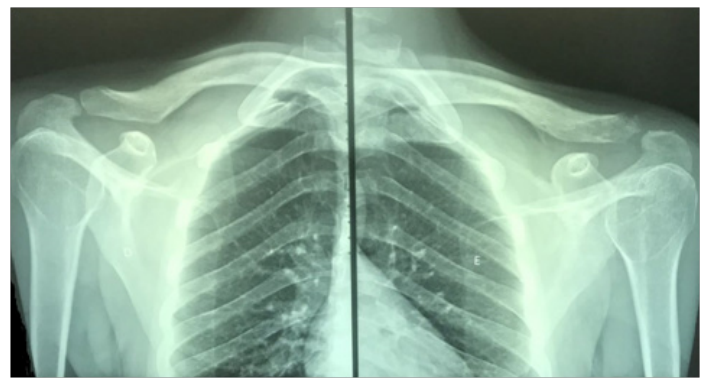

Figure 3 Post operative radiograph. Left: normal shoulder. Right: operated shoulder.

Among the cases with complications due to reduction loss, one was more evident, with severe vertical and horizontal instability. It is noteworthy that this patient did not follow postoperative guidance, not wearing the sling, nor completing the period for joint immobilization (Patient 4). Another complication we had was a case (Patient 1) of infection with positive cultures for $S$. aureus, with complete remission after antibiotic therapy and surgical debridement.

\section{Discussion}

The most common mechanism leading to acromioclavicular dislocation is direct force applied to the acromion with the joint in an adducted position, usually caused by a drop on the lateral side of the shoulder. ${ }^{1}$ This justifies the fact that the two main traumacausing events found in the present study were falls and motorcycle accidents, representing $55 \%$ and $45 \%$ of the cases, respectively. The latter may be explained by the large number of motorcycles existing in the state where our service operates, which, according to data from the Brazilian National Transport Confederation, is the highest among all the states in northeastern Brazil. Falling, on the other hand, may justify a higher age average of 42.75 years observed in our patients when compared to other studies, ${ }^{1,4,8,16-19}$ in which activities involving physical contact, such as sports, were more significant.

The majority of the evaluated patients were male $(85 \%)$, corroborating several studies. ${ }^{1,4,8,16-19}$ Such finding may be explained by the trauma mechanism, since men are more exposed to risky behavior. The modified Weaver-Dunn procedure is a widely used technique that involves coracoacromial ligament transfer and its insertion in the acromion toward the distal third of the clavicle with the aid of ties. ${ }^{11}$ Although excellent results have been reported with the use of this technique, there has also been evidence of some subluxation and complications in fixation. One of the reasons is that the resistance of the coracoacromial ligament comprises around $25 \%$ of the strength of the coracoclavicular ligaments, as shown by biomechanical studies. ${ }^{11,20}$ Besides, this non-anatomical reconstruction only guarantees coronal stability but does not correct the instability in the transverse or axial plane. ${ }^{14}$ One of the most positive points of this technique is the preservation of the acromioclavicular joint, since there is no thread penetration, with no need for another surgical intervention for suture removal.

Arliane et al..$^{21}$ in their study involving 122 orthopedic surgeons working in Brazil, evaluated the prevalence of several surgical techniques used for the treatment of these injuries. They evidenced that, among the acute injuries, $25.4 \%$ gave preference to the subcoracoid ties with acromioclavicular fixation, while $24.6 \%$ used only subcoracoid ties. Some of the complications associated with the first method include thread or endobutton migration and breakage of materials.

In the present study, we evaluated the functional outcomes based on the UCLA score, and all the results were classified as satisfactory, with $70 \%$ considered excellent, and $30 \%$, good. However, there was evidence of a high rate of reduction loss of the acromioclavicular joint, which corresponded to 6 of the 20 cases monitored. After an in-depth comparative functional analysis, we noticed that the average UCLA score after 25 weeks in the group presenting loss of reduction was 32 points, with five results considered good and one result classified as excellent. When evaluating the UCLA average after the same period in the 14 patients without loss of reduction, we noticed an average of 34.7 points, with 13 results considered excellent, and one result, good. There was evidence of a slight relationship between the functional and radiological aspects, without significantly compromising the overall functional result, since even in cases of subluxation as a complication, there was no dissatisfaction or limitation regarding the return to routine activities.

Some authors say that the loss of reduction is mainly due to the detachment or loosening of the threads from the clavicle, as well as the tear or rupture of the transferred coracoacromial ligament. ${ }^{16}$ It has been proved that the coracoacromial ligament is biomechanically inferior, and may lead to subluxation or chronical dislocation of the acromioclavicular joint in $30 \%$ of the cases. ${ }^{15}$

Nascimento et al. ${ }^{15}$ showed in their study that there was some reduction loss in all their cases when compared with the immediate postoperative period, which occurs around the 13th week. Only one case required a new approach due to symptomatology. Studies in the literature have shown that the loss of reduction does not affect the final clinical result of the treatment, as observed in our study. ${ }^{22-24}$

\section{Conclusion}

In the present study, we found that all the patients who underwent the described procedure presented satisfactory functional results (excellent and good), successfully returning to their everyday activities.

\section{Acknowledgments}

None.

\section{Conflicts of interest}

The authors declare no conflicts of interest in the development of this study.

\section{Funding}

None.

\section{References}

1. Rockwood CA, Green DP. Lesions of the acromioclavicular joint. In: Fractures in Adults.7th ed. Philadelphia: JB Lippincott; 2010. p. 1210 1242

2. Bishop JY, Kaeding C. Treatment of the acute traumatic acromioclavicular separation. Sports Med Arthrosc Rev. 2006;14(4):237-245.

3. Chillemi C, Franceschini V, Dei Giudici L, et al. Epidemiology of isolated acromioclavicular joint dislocation. Emerg Med Int. 2013;2013:171609. 
4. Babhulkar A, Pawaskar A. Acromioclavicular joint dislocations. Curr Ver Musculoskelet Med. 2014;7(1):33-39.

5. Saccomanno MF, DE Ieso C, Milano G. Acromioclavicular joint instability: anatomy, biomechanics, and evaluation. Joints. 2014;2(2):8792.

6. Faria RS, Ribeiro FR, Amin BO, et al. Acromioclavicular dislocation: postoperative evaluation of the coracoclavicular ligaments using magnetic resonance. Rev Bras Ortop. 2015;50(2):195-199.

7. Yoon JP, Lee BJ, Nam SJ, et al. Comparison of results between hook plate fixation and ligament reconstruction for acute unstable acromioclavicular joint dislocation. Clin Orthop Surg. 2015;7(1):97-103.

8. Takase K, Yamamoto K. Changes in surgical procedures for acromioclavicular joint dislocation over the past 30 years. Orthopedics. 2013;36(10):e1277-e1282.

9. De Carli A, Lanzetti RM, Ciompi A, et al. Acromioclavicular third degree dislocation: surgical treatment in acute cases. J Orthop Surg Res. 2015;10:13.

10. Tamaoki MJ, Belloti JC, Lenza M, et al. Surgical versus conservative interventions for treating acromioclavicular dislocation of the shoulder in adults. Cochrane Database Syst Rev. 2010;(8):CD007429.

11. Michlitsch MG, Adamson GJ, Pink M, et al. Biomechanical comparison of a modified Weaver-Dunn and a free-tissue graft reconstruction of the acromioclavicular joint complex. Am J Sports Med. 2010;38(6):11961203.

12. Moura DL, Reis AR, Ferreira $\mathrm{J}$, et al. A combined technique for acromioclavicular reconstruction after acute dislocation - technical description and functional outcomes. Rev bras ortop. 2018;53(1):67-74.

13. Weinstein DM, McCann PD, Mcllveen SJ, et al. Surgical Treatment of Complete Acromioclavicular Dislocations. Am J Sports Med. 1995;23(3):324-331.

14. Veado MA, Paiva AA, Pinto MD. Surgical treatment of complete acromioclavicular dislocation. Rev Bras Ortop. 2000;35(8):309-313.
15. Nascimento AT, Claudio GK. Functional and radiological evaluation of acute acromioclavicular dislocation treated with anchors without eyelet: comparison with other techniques. Rev bras ortop. 2016;51(5):561-568.

16. Scandiuzzi F, Torquato MT, Mizobuchi RR, et al. Surgical treatment of acromioclavicular dislocation by transferring the coracoacromial ligament. Rev Bras Ortop. 1999;34(5):305-312.

17. Fernandes MR, Fernandes RJ, Lech O. Grade III acromioclavicular dislocation: treatment using the Vukov technique. Rev Bras Ortop. 1995;30(9):669-673.

18. Simoni M, Brandão BL, Tumolo LH. Treatment of acromioclavicular dislocation with coracoclavicular ties by means of two mini-incisions. Rev Bras Ortop. 2005;40(8):483-489.

19. Morsy MG, Waly AH. Triple fixation for acute acromioclavicular joint dislocation. Egypt Orthop J. 2014;49(2):103-111.

20. Mazzocca AD, Arciero RA, Bicos J. Evaluation and treatment of acromioclavicular joint injuries. Am J Sports Med. 2007;35(2):316-329.

21. Arliani GG, Utino AY, Nishimura EM, et al. Acromioclavicular dislocation: treatment and rehabilitation. Current perspectives and trends among Brazilian orthopedists. Rev Bras Ortop. 2015;50(5):515-522.

22. Kovilazhikathu Sugathan H, Dodenhoff RM. Management of type 3 acromioclavicular joint dislocation: comparison of long-term functional results of two operative methods. ISRN Surg. 2012;2012:580504.

23. Beitzel K, Obopilwe E, Apostolakos J, et al. Rotational and translational stability of different methods for direct acromioclavicular ligament repair in anatomic acromioclavicular joint reconstruction. Am J Sports Med. 2014;42(9):2141-2148.

24. Beitzel K, Cote MP, Apostolakos J, et al. Current concepts in the treatment of acromioclavicular joint dislocations. Arthroscopy. 2013;29(2):387397. 